\title{
Rule-Based Technique for Monitoring of Voltage Events in Power Systems
}

\author{
Cheng-I Chen ${ }^{1, *}$, Yeong-Chin Chen ${ }^{2}$, and Chung-Hsien Chen $^{3}$ \\ ${ }^{1}$ Department of Electrical Engineering, National Central University, Taoyuan, Taiwan \\ ${ }^{2}$ Department of Computer Science and Information Engineering, Asia University, Taichung, Taiwan \\ ${ }^{3}$ Metal Industries Research and Development Centre, Taichung, Taiwan
}

\begin{abstract}
For the development of smart grid, the effective detection of voltage events is a very important task for the power system monitoring. Voltage sags, interruptions, and voltage swells which usually produce large losses in commercial and industrial consumers are the main events in power systems due to the sensitivity of equipments to these voltage variations. In this paper, a hybrid detection method for voltage events by combining the wavelet analysis and adaptive linear combiner (ADALINE) is proposed. The usefulness of the proposed algorithm is demonstrated by a simple laboratory setup with LabVIEW program and actual recorded waveforms. With the help of accurate time locating of the wavelet analysis and correct event classification of ADALINE, power system monitoring could provide accurate and useful information to power grids via the developing advanced metering infrastructure.
\end{abstract}

\section{Introduction}

With the development of energy policy, digital control, and system security, the traditional power system gradually can not provide the high power quality and system reliability. Therefore, the concept of smart grid has been proposed to meet these new requirements through integrated communications, advanced components, advanced control methods, sensing and measurement, and improved interfaces and decision support [1], [2].

Since the technology of advanced metering infrastructure (AMI) is the fundamental early step to grid modernization, the accurate and efficient monitoring of power quality and system states becomes a crucial task [3]. Among the numerous power quality disturbances, the voltage events are the most serious problems. Voltage sags, interruptions, and voltage swells which usually produce large losses in commercial and industrial consumers are the main events in power systems due to the sensitivity of equipments to these voltage variations. From the definition of IEEE Std. 1159-2009, the voltage sags are the sudden reductions (between $10 \%$ and $90 \%$ ) of the root-mean-square (RMS) voltage at a point in the electrical system [4]. The duration of the voltage sags is usually from 0.5 cycles to several seconds. The voltage sags may be caused by switching operations associated with a temporary disconnection of supply, the flow of heavy current associated with the start of large motor loads or the flow of fault currents. These events may come from customers' systems or from the supply network. On the contrary, the voltage swells are the brief increases in the
RMS voltage, which sometimes accompany with the voltage sags. Such phenomena usually appear on the unfaulted phases of a three-phase circuit that has developed a single-phase short circuit. When the load is suddenly rejected from the power system, the swells may also be present. The interruptions can be considered as the special case of voltage sags with almost $100 \%$ voltage drop. The cause of interruptions may be a blown fuse or breaker opening and leads to a significant shutdown. In order to prevent such effects, the analysis of voltage events and the development of strategies for protection of equipment have been of great concern in recent years.

In general, the analysis for the voltage events can be roughly divided into two categories: detection and classification, as shown in Fig. 1. The detection process is designed to identify the occurrences of events and trigger the corresponding automation and protection mechanisms. The classification process is mainly used to identify the types of events according to different properties of power quality disturbances.

According to IEEE Std. 1159-2009, the RMS magnitude of voltage supply is the standard method to detect and characterize the voltage events. The voltage events can be detected after a period of window data has been processed [5]. However, if the sampling rate could not make the number of samples per fundamental cycle integral, rounding the analysis window off would cause the estimation errors. Besides, the short time Fourier transform is another commonly used technique to track the RMS magnitude of voltage by introducing the shorter analysis window [6]. Although the detection with RMS values of voltage supply is a simple and easy-toimplement approach, it is not able to realize the points

Corresponding author: cichen@ee.ncu.edu.tw 


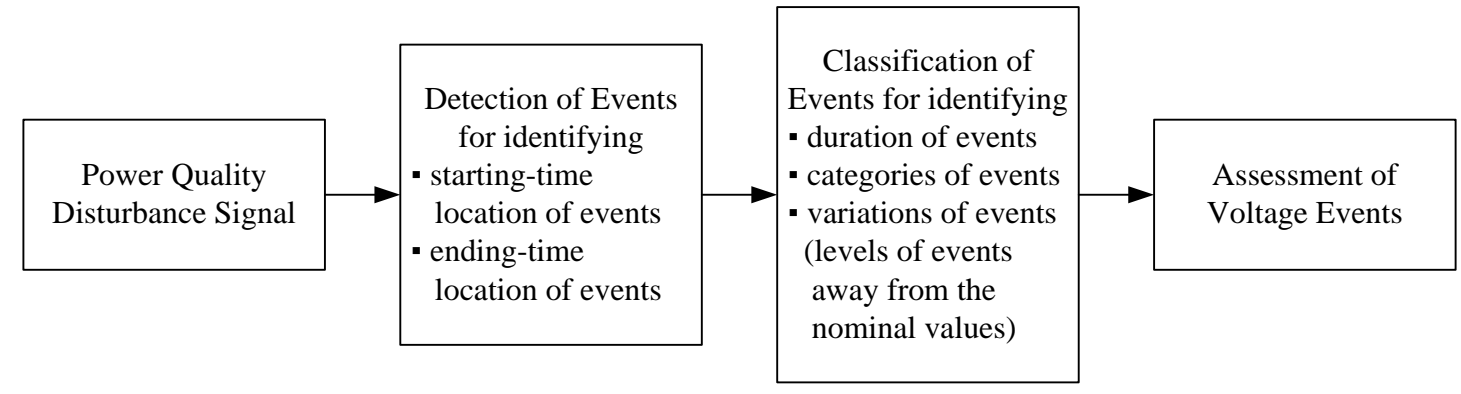

Fig. 1. Analysis procedure of voltage events.

on the power signal where the voltage event starts and ends. This limitation would deteriorate the estimation of event durations and lead to incorrect operations of protective devices.

Because the window-based methods are suitable for the analysis of voltage events rather than detection, some adaptive filtering techniques are proposed to solve this problem. A phase-locked loop based method is implemented for the detection of voltage sags in the realtime manner [7]. The automatic detection and analysis of voltage events are made through the Kalman filter-based method [8]. A fuzzy expert system based on ADALINE is used for the classification of power system disturbances [9]. However, these adaptive approaches always suffer from the numerical stability and computational burden. In addition, the problem of convergence in the detection procedure would make identification of time when events start and end difficult.

In recent years, the wavelet analysis has been proved to be a useful technique for the detection of voltage events [10]-[15]. When the event starts or ends, significant singular points often associated with sudden changes in power systems are present. The wavelet analysis provided the multi-resolution is a powerful tool for the locating of the singular signals. With the dyadic analysis structure of wavelet, the singular signals can be separated from those normal ones. Though the wavelet analysis only could achieve the detection of votlage events, identification of events needs to be further processed. Therefore, many advanced methods are proposed to solve this problem, such as neural network, fuzzy, and hidden Markov model. However, these techniques usually take many computational operations and need data training.

In this paper, a hybrid detection method for voltage events by combining the wavelet analysis and ADALINE is proposed. Different from the windowbased calculations in the standard RMS method, the ADALINE method could track the signal recursively. Since the estimation model in the ADALINE is expressed with the Fourier series, the instantaneous RMS value at each time instant can be easily obtained via the gradient descent method. With the help of accurate time locating of the wavelet analysis and correct event classification of ADALINE, power system monitoring could provide accurate and useful information to power grids via the developing AMI. The organization of this paper is as follows. In section 2, the hybrid technique for the analysis of voltage events is proposed. To verify the performance of proposed method, a design of the virtual instrument is built and some comprehensive case studies are investigated in section 3 .

\section{Proposed hybrid method}

To characterize voltage disturbances, sinusoidal models are the most suitable ones. For a measured signal $y$, its discrete-time form, $y(n)$, of finite length $N$ sampled the time interval $\Delta t$ can be represented by $H$ sinusoidal components with noises as

$$
y(n)=\sum_{h=1}^{H} a_{h} \cos \left(n \omega_{h} \Delta t+\phi_{h}\right)+w(n)
$$

where ${ }^{a_{h}}$ is the amplitude, $\phi_{h}$ is the initial phase angle, $\omega_{h}=h \times 2 \pi f_{1}$ is the radian harmonic frequency of $h^{\text {th }}$ harmonic component, and $w(n)$ represents the additive white noise. For the analysis of voltage events, the harmonic components are usually ignored, as given in (2).

$$
y(n)=a_{1} \cos \left(\omega_{1} \Delta t+\phi_{1}\right)+w(n)
$$

For discussing the performance of analysis methods, wavelet analysis and ADALINE are firstly reviewed. Then, the hybrid method by combining the advantages of these two approaches is introduced.

\section{A. Wavelet Analysis}

Wavelet analysis is a powerful tool to provide a set of sub-band filters for the signal decomposition. With the characteristic of the short-duration oscillating waveforms with zero mean and fast decay to zero amplitude at both ends, the multiresolution analysis can be performed, as shown in Fig. 2 [11]-[14].

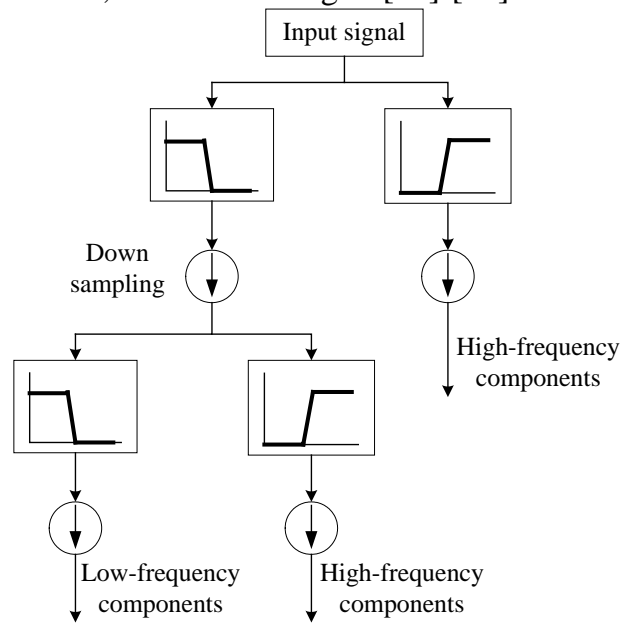

Fig. 2. Multiresolution decomposition structure of wavelet analysis.

*Corresponding author: cichen@ee.ncu.edu.tw 


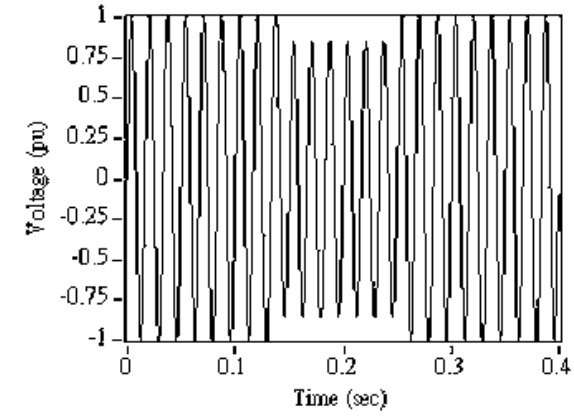

(a)

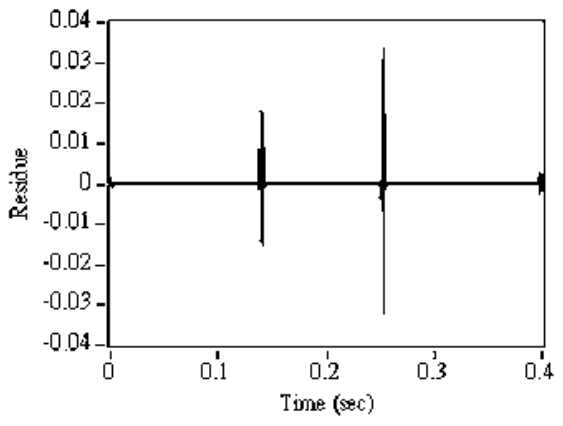

(d)

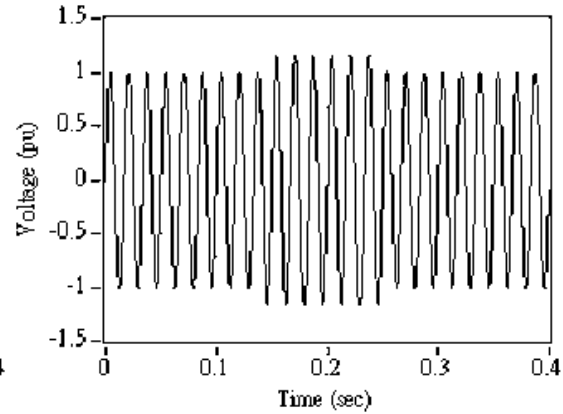

(b)

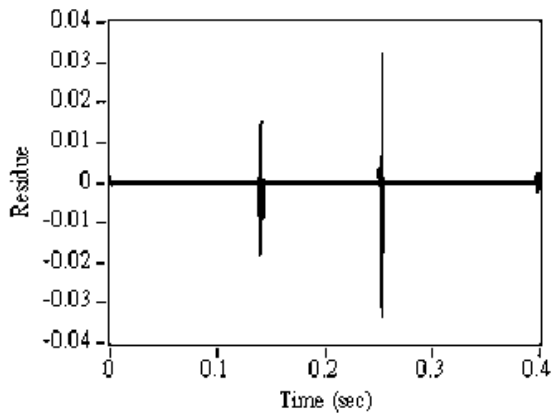

(e)



(c)

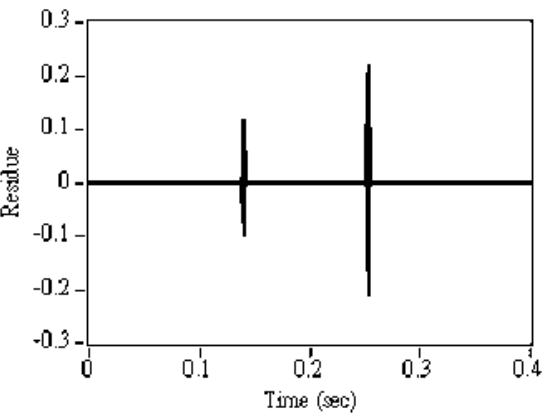

(f)

Fig. 3. Detection of voltage events with the wavelet analysis: (a) waveform of sag; (b) waveform of swell; (c) waveform of interruption; (d) detection result of sag; (e) detection result of swell; (f) detection result of interruption.

Without estimating the RMS value of the signal, such method performs the detection by observing the residual values between the analysis model and the actual signal. When the event starts or ends, the singular signal samples would cause significant estimation errors in the analysis model. Therefore, the time location of events can be detected more accurately than the traditional window-based methods and adaptive filtering techniques. For the signals of voltage sag, swell, and interruption, it is easily found that the residual method can effectively detect the starting and ending time of the events, as shown in Fig. 3. However, the categories of voltage events are not able to recognize. Therefore, it is necessary to find an efficient approach to solve this problem.

\section{B. ADALINE Detection Method}

ADALINE is an adaptive filter used for noise canceling or signal extraction. In recent years, this method has been widely used in the area of power quality studies. ADALINE is generally used for the harmonic analysis by expressing the discrete form of the estimated signal $y$ in (1) as

$$
\begin{aligned}
& y(n) \\
= & \sum_{m=1}^{M} a_{m} \cos \left(2 \pi m f_{1} n \Delta t+\phi_{m}\right) \\
= & \sum_{m=1}^{M}\left(a_{m} \cos \phi_{m} \cos 2 \pi m f_{1} n \Delta t-a_{m} \sin \phi_{m} \sin 2 \pi m f_{1} n \Delta t\right) \\
= & \sum_{m=1}^{M}\left(w_{2 m-1} \cos \theta_{m}-w_{2 m} \sin \theta_{m}\right)
\end{aligned}
$$

where $M$ is the estimation order represented the number of considered harmonics, $\theta_{m}=2 \pi f_{1} m n \Delta t$, $w_{2 m-1}=a_{m} \cos \phi_{m}$, and $w_{2 m}=a_{m} \sin \phi_{m}$. Equation (3) can be rewritten as

$$
y(n)=\mathbf{w}^{T}(n) \cdot \mathbf{x}(n)
$$

where

$$
\mathbf{w}(n)=\left[\begin{array}{c}
w_{1} \\
w_{2} \\
\vdots \\
w_{2 M-1} \\
w_{2 M}
\end{array}\right] \text { and } \mathbf{x}(n)=\left[\begin{array}{c}
\cos \theta_{1} \\
-\sin \theta_{1} \\
\vdots \\
\cos \theta_{M} \\
-\sin \theta_{M}
\end{array}\right]
$$

are weight and input vectors of ADALINE, respectively. By minimizing the estimation error with the gradient descent method, the amplitude and phase angle of the $m^{\text {th }}$ harmonic are obtained by calculating

$$
\begin{aligned}
& a_{m}=\sqrt{w_{2 m-1}^{2}+w_{2 m}^{2}} \\
& \phi_{m}=\tan ^{-1}\left(\frac{w_{2 m}}{w_{2 m-1}}\right)
\end{aligned}
$$

Since the harmonic components are usually ignored in the analysis of voltage events, the estimation order $M$ would be simplified to be one. Due to the adaptive solution procedure in such detection mechanism, the convergence associated with the learning parameters (step sizes) would interfere with the accuracy of time locating for the voltage events.

When the value of learning parameter is higher than the suitable one, the detection result would cause the overshooting effect, as shown in Figs. 4(a)-(c). Once the value of learning parameter is set to be lower, the

'Corresponding author: cichen@ee.ncu.edu.tw 


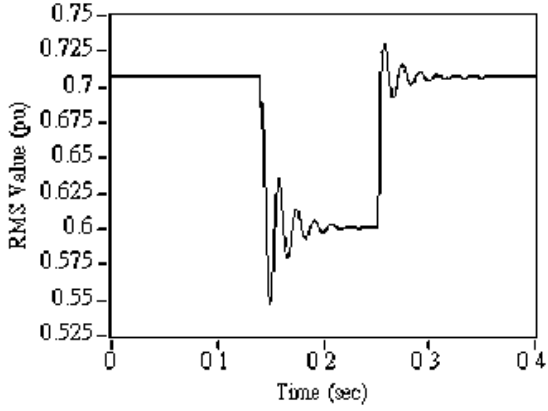

(a)

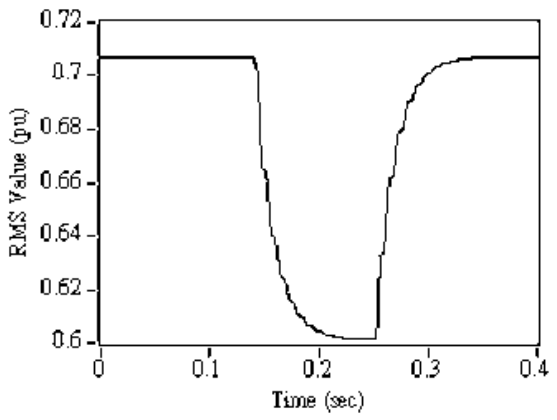

(d)

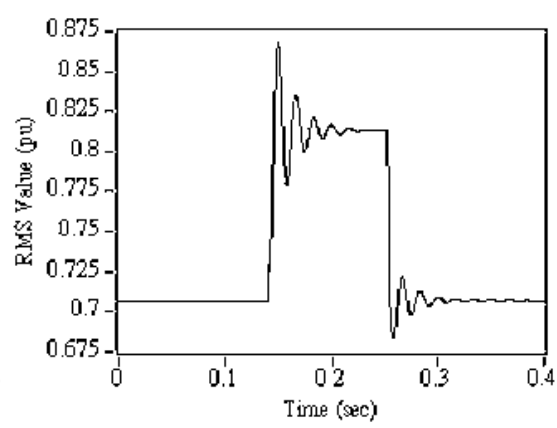

(b)

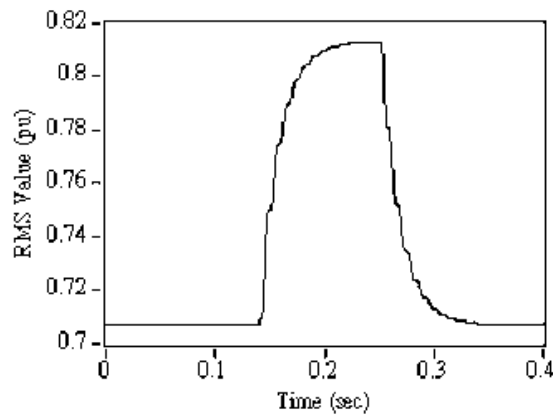

(e)

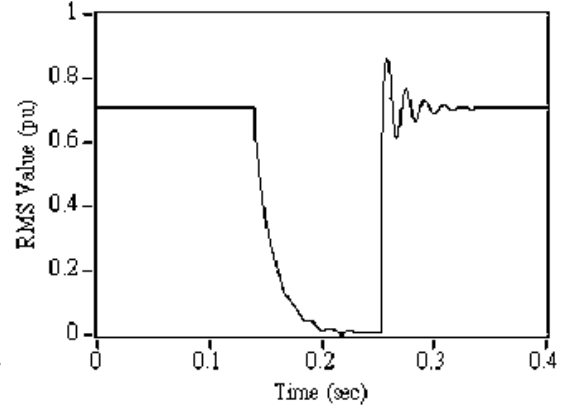

(c)

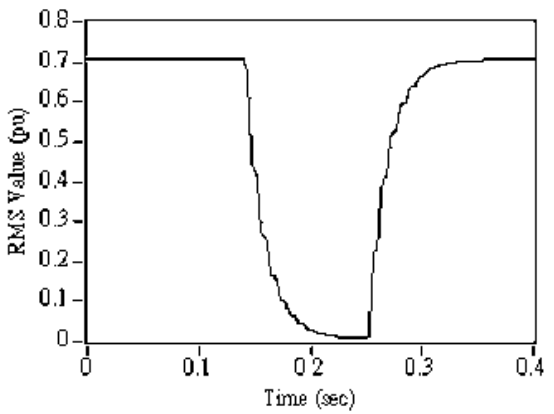

(f)

Fig. 4. Detection of voltage events with ADALINE detection method: (a) detection result with overshooting effect for sag in Fig. 3(a); (b) detection result with overshooting effect for swell in Fig. 3(b); (c) detection result with overshooting effect for interruption in Fig. 3(c); (d) detection result with low numerical convergence for sag in Fig. 3(a); (e) detection result with low numerical convergence for swell in Fig. 3(b); (f) detection result with low numerical convergence for interruption in Fig. 3(c).

duration in Figs. 4(d)-(f) to achieve the numerical convergence may take more time. Such effect would deteriorate the time resolution for the locating of events. The same characteristic of ADALINE with traditional RMS estimation method is that the determination of threshold for the event classification is simple and convenient according to the definition in IEEE Std. 1159-2009, as given in Table 1 [4].

Table 1. Categories and typical characteristics of sags, swells, and interruptions [4]

\begin{tabular}{|c|c|c|c|}
\hline \multicolumn{2}{|c|}{ Categories } & Typical duration & $\begin{array}{c}\text { Typical voltage } \\
\text { magnitude }\end{array}$ \\
\hline \multirow{8}{*}{$\begin{array}{l}\text { Short duration } \\
\text { variations }\end{array}$} & Instantaneous Sag & 0.5 - 30 cycles & $0.1-0.9 \mathrm{pu}$ \\
\hline & $\begin{array}{c}\text { Instantaneous } \\
\text { Swell }\end{array}$ & 0.5 - 30 cycles & $1.1-1.8 \mathrm{pu}$ \\
\hline & $\begin{array}{l}\text { Momentary } \\
\text { Interruption }\end{array}$ & 0.5 cycles -3 sec & $<0.1 \mathrm{pu}$ \\
\hline & Momentary Sag & 30 cycles -3 sec & $0.1-0.9 \mathrm{pu}$ \\
\hline & Momentary Swell & 30 cycles -3 sec & $1.1-1.4 \mathrm{pu}$ \\
\hline & $\begin{array}{l}\text { Temporary } \\
\text { Interruption }\end{array}$ & $3 \mathrm{sec}-1 \mathrm{~min}$ & $<0.1 \mathrm{pu}$ \\
\hline & Temporary Sag & $3 \mathrm{sec}-1 \mathrm{~min}$ & $0.1-0.9 \mathrm{pu}$ \\
\hline & Temporary Swell & $3 \mathrm{sec}-1 \mathrm{~min}$ & $1.1-1.2 \mathrm{pu}$ \\
\hline \multirow{3}{*}{$\begin{array}{l}\text { Long duration } \\
\text { variations }\end{array}$} & $\begin{array}{l}\text { Interruption, } \\
\text { sustained }\end{array}$ & $>1 \min$ & $0.0 \mathrm{pu}$ \\
\hline & Undervoltages & $>1 \mathrm{~min}$ & $0.8-0.9 \mathrm{pu}$ \\
\hline & Overvoltages & $>1$ min & $1.1-1.2 \mathrm{pu}$ \\
\hline
\end{tabular}

\section{Proposed Hybrid Method}

Since the residual method only can determine the time positions of event appearances, the classification method is usually necessary to make the power quality analysis. The artificial neural network (ANN), fuzzy theory, and the clustering method are the commonly used techniques foe event classification. In general, these methods are based on the pattern identification.
Therefore, the data for the training process are usually necessary. The training data can be obtained directly with the power quality disturbances or with the features from the detection methods. However, the performance of classification is usually dependent on the extracted features. In addition, the speed of convergence and accuracy of classification in the ANN-based schemes are affected by the signal-to-noise ratio. Therefore, the definitions of dominant features, sizes of network or clustering, threshold values for training and condition of convergence, structures of classification algorithms, and updating approaches for the training process are the important factors to the performance of classification and make the classification more complex and difficult to debug.

The rule-based methods have attracted much attention in recent years [16]. The usage of rule-based methods could reduce the difficulty of modeling and analysis of complicated systems. Rule-based methods are suitable for incorporating the qualitative aspects of human experience within the mapping laws [14]. However, these methods usually perform the classification of events based on the detection features empirically, such as the fundamental component, phase angle shift, total harmonic distortion, number of peaks of wavelet coefficients, energy of wavelet coefficients, and lower harmonic distortion [16]. To facilitate the determination of triggering thresholds for the classification, the direct usage of quantities listed in Table I is the most convenient and practicable way. Therefore, a hybrid method combined with ADALINE and the wavelet analysis is proposed in this paper to provide the accurate disturbance analysis. 
Figure 5 represents the proposed hybrid analysis process for detecting the voltage events. If the voltage events are present, the detection of wavelet analysis will be activated to perform the time locating of disturbances. Once the triggering threshold is met, the analyzed RMS magnitudes of ADALINE $A_{\text {event }}$ within the duration of events $D_{\text {event }}$ will be extracted. Then, the classification can be performed by comparing the extracted information with the conditional rules listed in the following.

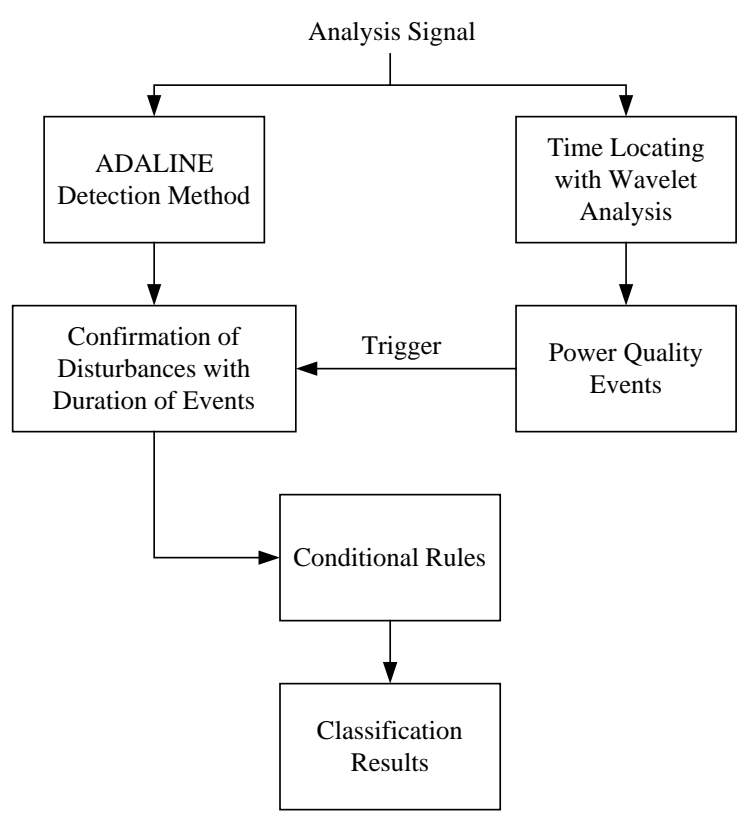

Fig. 5. Proposed analysis process for the voltage events.

\section{Rule 1:}

\section{If}

$1.1 \mathrm{pu}>\max \left\{A_{\text {event }}(X), X \in\right.$ sample set within $\left.D_{\text {event }}\right\}$ and

$\min \left\{A_{\text {event }}(X), X \in\right.$ sample set within $\left.D_{\text {event }}\right\} \geq 0.9 \mathrm{pu}$, then the classification of such event is normal.

Rule 2:

If $\quad 0.9 \mathrm{pu}>\max \left\{A_{\text {event }}(X), X \in\right.$ sample set within $\left.D_{\text {event }}\right\}$ $\min \left\{A_{\text {event }}(X), X \in\right.$ sample set within $\left.D_{\text {event }}\right\} \geq 0.1$ pu , and 30 cycles $\geq D_{\text {event }}>0.5$ cycles, then the classification of such event is instantaneous sag.

Rule 3: If $\quad 0.9 \mathrm{pu}>\max \left\{A_{\text {event }}(X), X \in\right.$ sample set within $\left.D_{\text {event }}\right\}$ and $D_{\text {event }}>1 \mathrm{~min}$, then the classification of such event $\min \left\{A_{\text {event }}(X), X \in\right.$ sample set within $\left.D_{\text {event }}\right\} \geq 0.1$ pu , and 3 sec $\geq D_{\text {event }}>30$ cycles, then the classification of such event is momentary sag.

\section{Rule 4:}

If $\quad 0.9 \mathrm{pu}>\max \left\{A_{\text {event }}(X), X \in\right.$ sample set within $\left.D_{\text {event }}\right\}$, next trigger.

$\min \left\{A_{\text {event }}(X), X \in\right.$ sample set within $\left.D_{\text {event }}\right\} \geq 0.1 \mathrm{pu}$, and $1 \mathrm{~min} \geq D_{\text {event }}>3 \mathrm{sec}$, then the classification of such event is temporary sag.

\section{Rule 5:}

If $\quad 0.9 \mathrm{pu}>\max \left\{A_{\text {event }}(X), X \in\right.$ sample set within $\left.D_{\text {event }}\right\}$, $\min \left\{A_{\text {event }}(X), X \in\right.$ sample set within $\left.D_{\text {event }}\right\} \geq 0.8 \mathrm{pu}$, and $D_{\text {event }}>1 \mathrm{~min}$, then the classification of such event is undervoltage.

\section{Rule 6:}

If $\quad 1.8 \mathrm{pu}>\max \left\{A_{\text {event }}(X), X \in\right.$ sample set within $\left.D_{\text {event }}\right\}$, $\min \left\{A_{\text {event }}(X), X \in\right.$ sample set within $\left.D_{\text {event }}\right\} \geq 1.1$ pu , and 30 cycles $\geq D_{\text {event }}>0.5$ cycles, then the classification of such event is instantaneous swell.

\section{Rule 7:}

If $\quad 1.4 \mathrm{pu}>\max \left\{A_{\text {event }}(X), X \in\right.$ sample set within $\left.D_{\text {event }}\right\}$, $\min \left\{A_{\text {event }}(X), X \in\right.$ sample set within $\left.D_{\text {event }}\right\} \geq 1.1$ pu , and $3 \mathrm{sec} \geq D_{\text {event }}>30$ cycles, then the classification of such event is momentary swell.

\section{Rule 8:}

If $\quad 1.2 \mathrm{pu}>\max \left\{A_{\text {event }}(X), X \in\right.$ sample set within $\left.D_{\text {event }}\right\}$, $\min \left\{A_{\text {event }}(X), X \in\right.$ sample set within $\left.D_{\text {event }}\right\} \geq 1.1$ pu , and $1 \mathrm{~min} \geq D_{\text {event }}>3 \mathrm{sec}$, then the classification of such event is temporary swell.

Rule 9:

If $\quad 1.2 \mathrm{pu}>\max \left\{A_{\text {event }}(X), X \in\right.$ sample set within $\left.D_{\text {event }}\right\}$, $\min \left\{A_{\text {event }}(X), X \in\right.$ sample set within $\left.D_{\text {event }}\right\} \geq 1.1$ pu , and $D_{\text {event }}>1 \mathrm{~min}$, then the classification of such event is overvoltage.

\section{Rule 10:}

If $\quad 0.1 \mathrm{pu}>\max \left\{A_{\text {event }}(X), X \in\right.$ sample set within $\left.D_{\text {event }}\right\}$, and $3 \mathrm{sec} \geq D_{\text {event }}>0.5$ cycles, then the classification of such event is momentary interruption.

\section{Rule 11:}

If $\quad 0.1 \mathrm{pu}>\max \left\{A_{\text {event }}(X), X \in\right.$ sample set within $\left.D_{\text {event }}\right\}$, and $1 \mathrm{~min} \geq D_{\text {event }}>3 \mathrm{sec}$, then the classification of such event is temporary interruption.

\section{Rule 12:}

If $\max \left\{A_{\text {event }}(X), X \in\right.$ sample set within $\left.D_{\text {event }}\right\} \approx 0$, is interruption, sustained.

\section{Rule 13:}

If $D_{\text {event }} \leq 0.5$ cycles, then such event is falsely triggered. Therefore, this false trigger would be discarded and the process would keep waiting for the

It is observed that the threshold quantities for the event identification are determined according to IEEE Std. 1159-2009. The traditional problem of the residual detection methods, which is unable to identify the types 
of events, can be resolved with ADALINE. Other advantages of proposed solution mechanism are that the calculations of specially defined features and training of classification are not required. Therefore, the computational burden can be reduced and the complex determination of related parameters for the training is not required.

\section{Design of virtual instrument and case studies}

To verify the performance of proposed hybrid analysis method for voltage events, a virtual instrument implemented with LabVIEW is developed. The graphical user interface of this analysis system is shown in Fig. 6. After setting some parameters of system (i.e. sampling frequency, number of extracted data samples, etc.) in block $A$, the analysis of voltage events would be activated by clicking the button of block B. Then, the extracted waveform of power signal would be displayed in block C. With the help of wavelet analysis, the time locating of voltage events can be determined (block D). Simultaneously, the ADAINE detection method (block F) performs the analysis for the power signal in block $C$. By capturing the detected RMS value within the duration of voltage event (block E), the identification of event categories can be performed with the conditional rules addressed in section II-C and the result will be shown in block G. Finally, the users of this system could save the analysis results, including waveform of analyzed signal, locations of voltage event, detected RMS value, and category of event, by clicking the button of block $\mathrm{H}$. In the following, several actual voltage events extracted from substation of some power company are tested.

\section{A. Detection of Sag}

In the first case study, a voltage sag event is detected through the wavelet analysis. Since the wavelet analysis is sensitive to the noise, the residual coefficient of wavelet is enhanced to help trigger of voltage event by using the squaring method [17]. The reason is that the singular points are high-frequency components when the voltage events are present, so the information about these points is decomposed into the detail coefficients of high-frequency components in Fig. 2. In general, the coefficients related to the singular signals are more dominant than those with respect to the noise. Therefore, the time locations of the events can be enhanced by directly squaring the detail coefficients. From the detection result of ADALINE, it is easily observed that this power signal contains the voltage sag of $25 \%$, as shown in Fig. 7

\section{B. Detection of Swell}

In the second case study, a 0.2 -sec waveform with approximately 2.5-cycle voltage swell is investigated. The starting and ending time of this voltage event can be easily detected via the wavelet analysis. With the help of analysis through ADALINE, the category of event can be determined. Since the duration of event is approximate 2.5 cycles and the analyzed signal is with the voltage swell of $16 \%$, it is convenient to identify that the waveform in Fig. 8(a) is an instantaneous swell, according to Rule 6 addressed in section 2-C and Table 1.

\section{Detection of Interruption}

For the final case study, a voltage interruption of approximately 2.5 cycles is present in the analyzed signal. According to the detection of the wavelet analysis, the duration within the starting and ending time would be used to help ADALINE identify the category of event. From Figs. 9(a) and 9(b), it is found that the duration of event is approximately 2.5 cycles and the magnitude of voltage varies to $0.02 \mathrm{pu}$. As a result, this power signal can be classified as the momentary interruption based on the definition in Table 1 and Rule 10.

\section{Conclusions}

In this paper, an efficient and useful method combined with the wavelet analysis and ADALINE is proposed for the analysis of voltage events. With the multi-resolution of wavelet transform, the time locating of voltage events can be easily determined. The drawback unable to recognize the category of event in the wavelet analysis has been solved with the combination of ADALINE. The first benefit of this proposed analysis mechanism is that the training of patterns for the voltage events is not necessary. In addition, the empirical determination of detection features for the classification of events can be prevented. Finally, the rules for the event classification can be conveniently adjusted according to the revision of international standard. With the development of smart grid, the proposed analysis technique for voltage events can be used as a useful reference to the set-up of AMI. Since the wavelet analysis is sensitive to the noise, a more robust approach rather than the squaring method may be necessary for the future work.

\section{References}

1. National Energy Technology Laboratory for the U.S. Department of Energy Office of Electricity Delivery and Energy Reliability, "The NETL Modern Grid Initiative - A Vision for the Modern Grid,” March 2007.

2. National Energy Technology Laboratory for the U.S. Department of Energy Office of Electricity Delivery and Energy Reliability, "NETL Modern Grid Initiative Powering our $21^{\text {st }}$-Century Economy - A Compendium of Modern Grid Technologies,” June 2007.

3. National Energy Technology Laboratory for the U.S. Department of Energy Office of Electricity Delivery and Energy Reliability, "NETL Modern Grid Strategy Powering our 21 $1^{\text {st }}$-Century Economy Advanced Metering Infrastructure,” Feb. 2008. 
power quality, IEEE Std. 1159-2009, Jun. 2009. System Configuration (A)

Save Analysis Results (H)

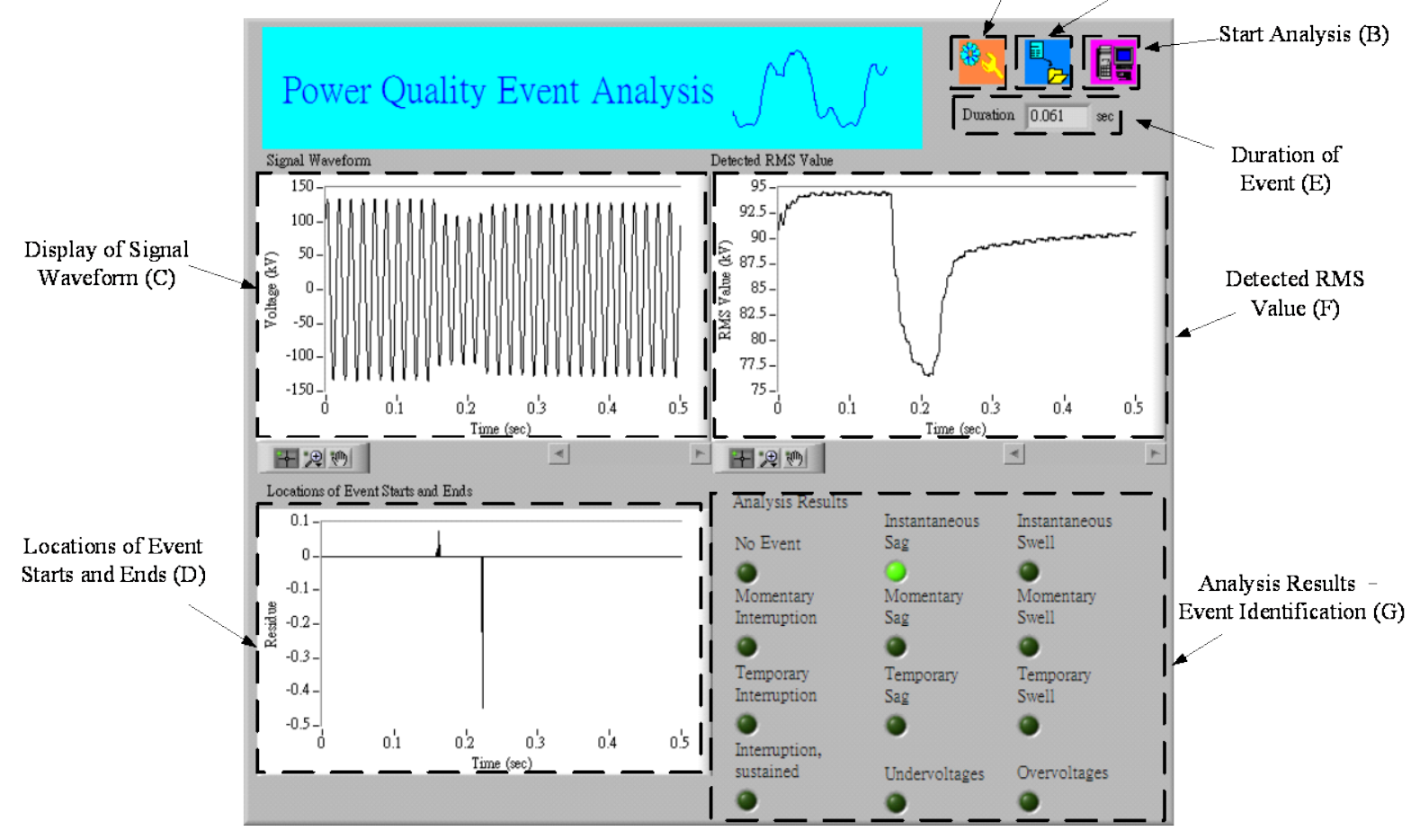

Fig. 6. Graphical user interface of virtual instrument for the analysis of voltage events.

5. T. Radil, P. M. Ramos, F. M. Janeiro, and A. C. Serra, "PQ Monitoring System for Real-Time Detection and Classification of Disturbances in a Single-Phase Power System," IEEE Trans. on Instrumentation and Measurement, Vol. 57, No. 8, Aug. 2008, pp. 1725-1733.

6. Y. H. Gu and M. H. J. Bollen, "Time-Frequency and Time-Scale Domain Analysis of Voltage Disturbances," IEEE Trans. on Power Delivery, Vol. 15, No. 4, Oct. 2000, pp. 1279-1284.

7. R. Naidoo and P. Pillay, "A New Method of Voltage Sag and Swell Detection,” IEEE Trans. on Power Delivery, Vol. 22, No. 2, April 2007, pp. 1056-1063.

8. J. Barros and E. Perez, "Automatic Detection and Analysis of Voltage Events in Power Systems," IEEE Trans. on Instrumentation and Measurement, Vol. 55, No. 5, Oct. 2006, pp.1487-1493.

9. P. K. Dash, S. Mishra, M. M. A. Salama, and A. C. Liew, "Classification of Power System Disturbances Using a Fuzzy Expert System and a Fourier Linear Combiner," IEEE Trans. on Power Delivery, Vol. 15, No. 2, April 2000, pp. 472-477.

10. T. K. Abdel-Galil, E. F. El-Saadany, A. M. Youssef, M. M. A. Salama, "Disturbance Classification Using Hidden Markov Models and Vector Quantization,” IEEE Trans. on Power Delivery, Vol. 20, No. 3, July 2005, pp. 2129-2135.

11. J. Huang, M. Negnevitsky, and D. T. Nguyen, "A Neural-Fuzzy Classifier for Recognition of Power Quality Disturbances," IEEE Trans. on Power Delivery, Vol. 17, No. 2, April 2002, pp. 609-616.
12. H. He and J. A. Starzyk, “A Self-Organizing Learning Array System for Power Quality Classification Based on Wavelet Transform,” IEEE Trans. on Power Delivery, Vol. 21, No. 1, Jan. 2006, pp. 286-295.

13. P. Janik and T. Lobos, "Automated Classification of Power-Quality Disturbances Using SVM and RBF Networks,” IEEE Trans. on Power Delivery, Vol. 21, No. 3, July 2006, pp. 1663-1669.

14. M. B. I. Reaz, F. Choong, M. S. Sulaiman, F. MohdYasin, and M. Kamada, "Expert System for Power Quality Disturbance Classifier," IEEE Trans. on Industrial Electronics, Vol. 22, No. 3, July 2007, pp. 1979-1988.

15. I. Monedero, C. Leon, J. Ropero, A. Garcia, J. M. Elena, and J. C. Montano, "Classification of Electrical Disturbances in Real Time Using Neural Networks,” IEEE Trans. on Power Delivery, Vol. 22, No. 3, July 2007, pp. 1288-1296.

16. M. Kezunovic and Y. Liao, "A novel software implementation concept for power quality study," IEEE Trans. on Power Delivery, Vol. 17, No. 2, April 2002, pp. 544-549.

17. S. Santoso, E. J. Powers, W. Mack Grady, and Peter Hofmann, "Power quality assessment via wavelet transform analysis,” IEEE Trans. on Power Delivery, Vol. 11, No. 2, Apr. 1996, pp. 924-930.

\section{Acknowledgement}

The authors would like to acknowledge the financial support by the Ministry of Science and Technology of 
Taiwan, Republic of China, under Grants MOST 1072628-E-008 -002 -MY3 and MOST 107-3113-E-008003- for this work.

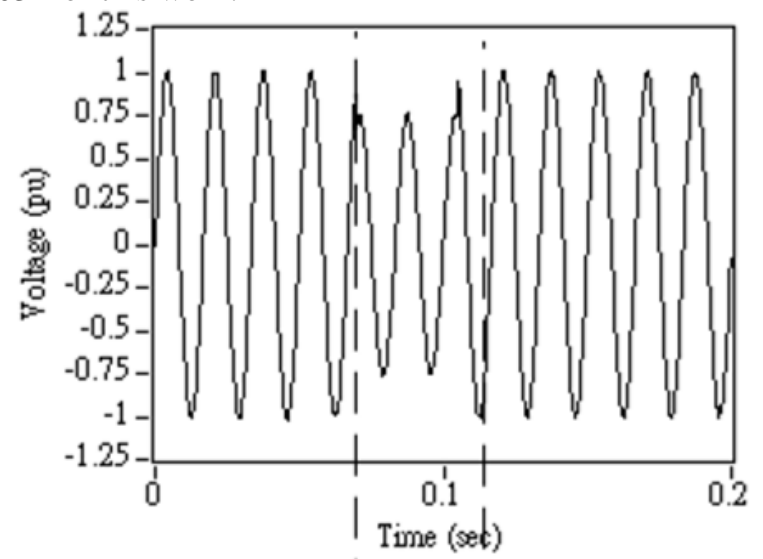

(a)

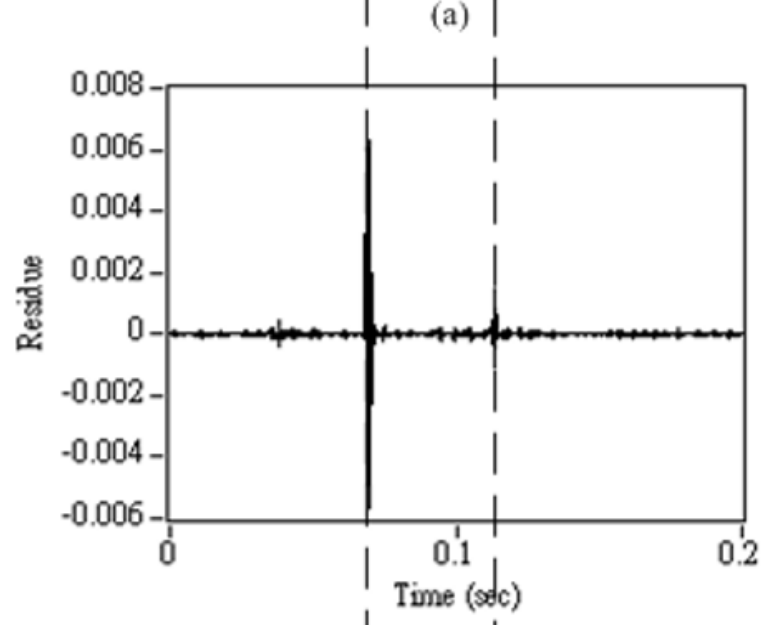

(b)

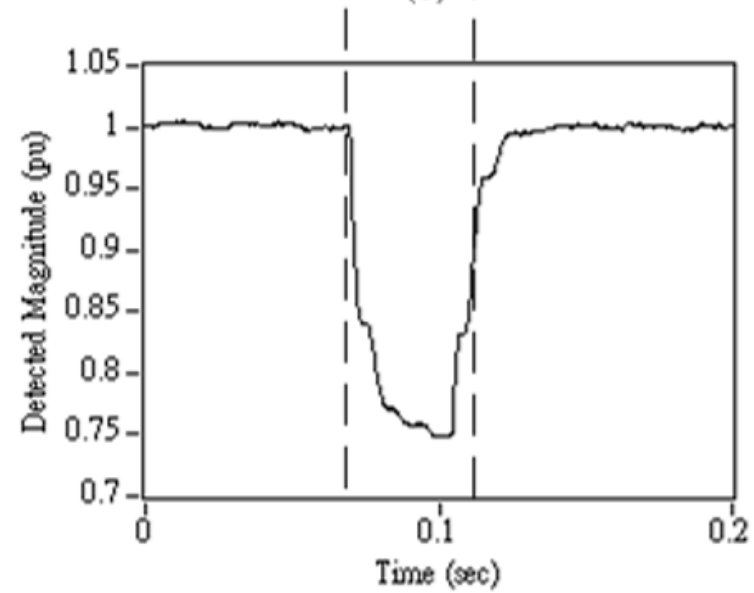

(c)

Fig. 7. (a) Waveform of the captured voltage sag. (b) Detection result of wavelet analysis. (c) Detected magnitude of ADALINE.

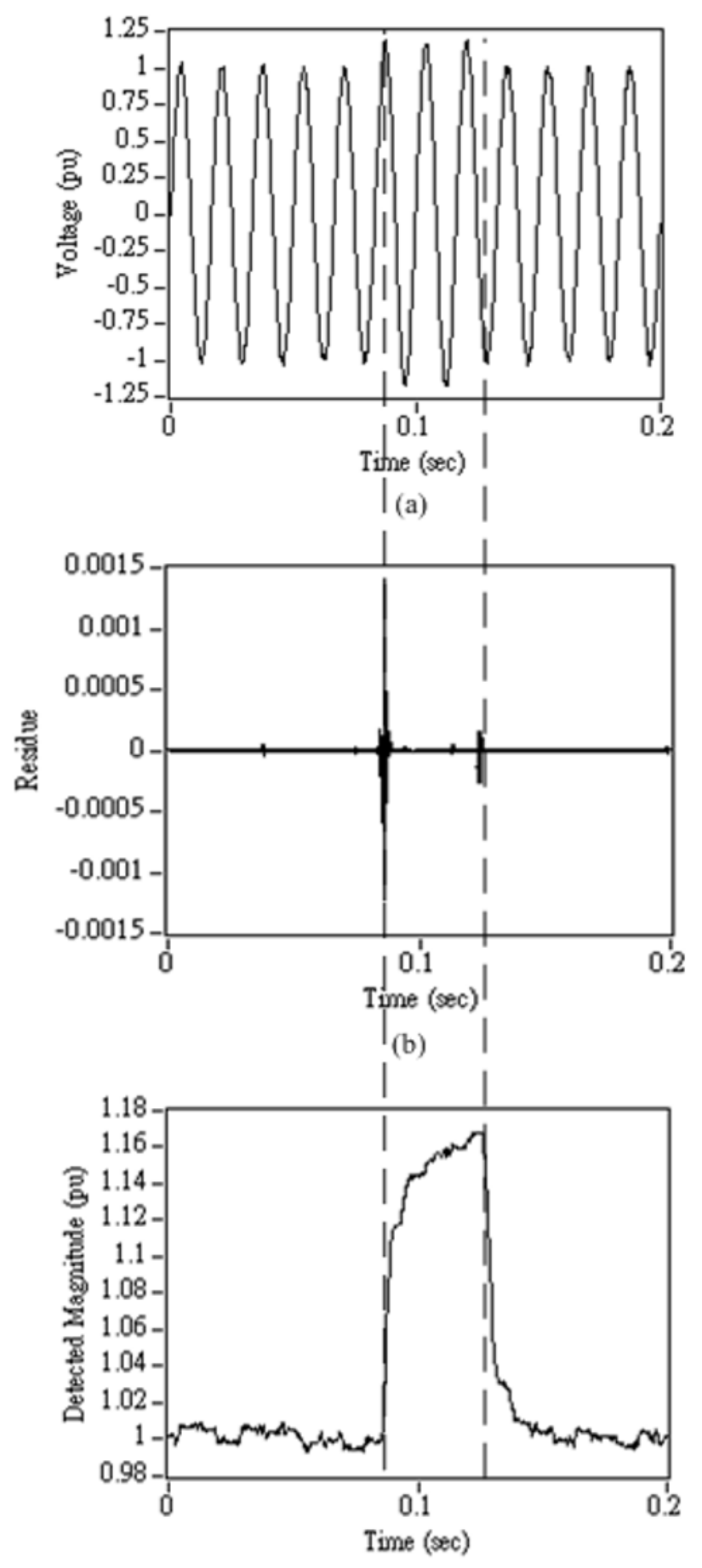

(c)

Fig. 8. (a) Waveform of the captured voltage swell. (b) Detection result of wavelet analysis. (c) Detected magnitude of ADALINE. 


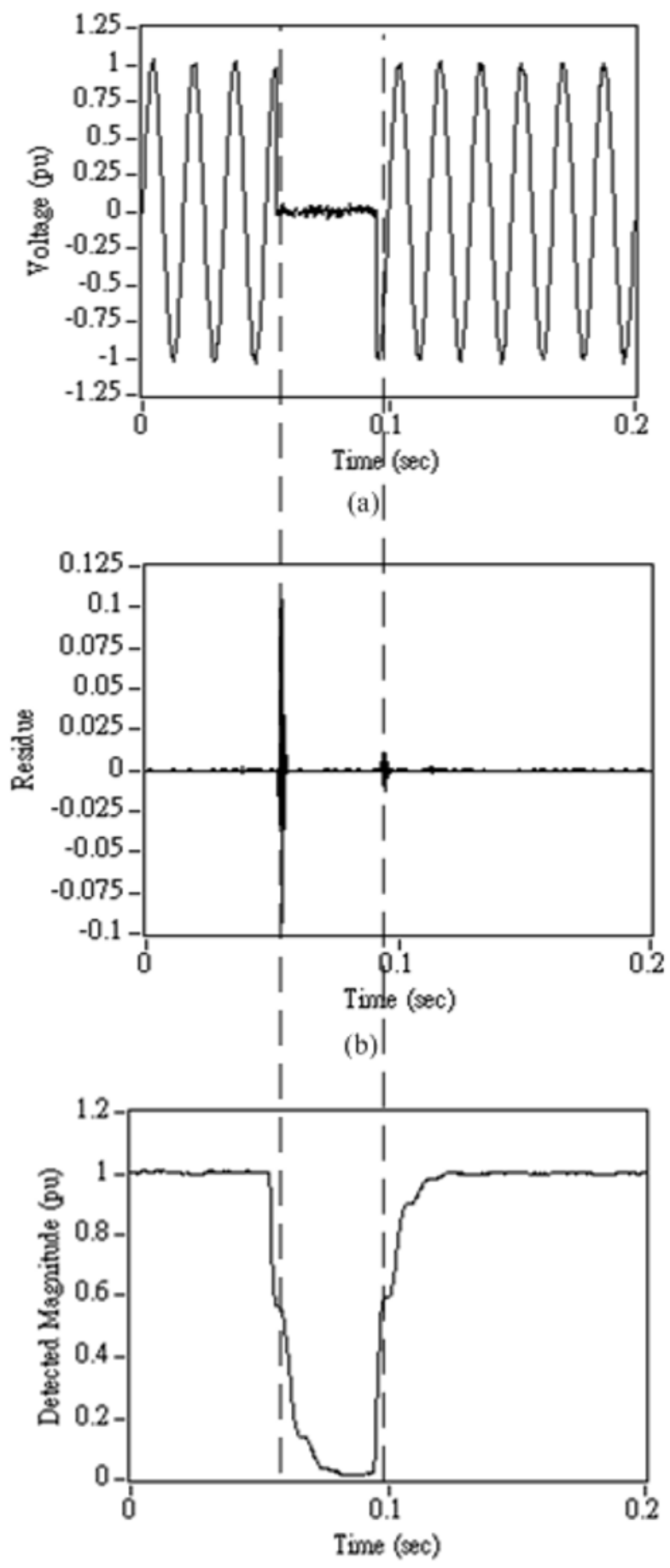

(c)

Fig. 9. (a) Waveform of the captured voltage interruption. (b) Detection result of wavelet analysis. (c) Detected magnitude of ADALINE. 